\title{
Boundary value problems for second order differential equations with $\varphi$-Laplacians
}

\author{
STANISŁAW SȨDZIWY (D
}

\begin{abstract}
A new method for solving the boundary value problems for the second order ODEs with bounded nonlinearities and singular $\varphi-$ Laplacians is presented.
\end{abstract}

Mathematics Subject Classification. Primary: 34B16; Secondary: 34B15.

Keywords. Nonlinear boundary value problem, Singular $\phi$-Laplacian, Borsuk's theorem.

1. Introduction. The paper gives a simple proof of the existence and multiplicities of solutions to the boundary value problems (BVPs)

$$
\begin{aligned}
& \left(\varphi\left(u^{\prime}\right)\right)^{\prime}=f\left(t, u, u^{\prime}\right)-s, \\
& L\left(u, u^{\prime}\right)=0,
\end{aligned}
$$

relative to the value of $s$, assuming that $\varphi:(-a, a) \rightarrow \mathbb{R}, \varphi(0)=0$, is an increasing homeomorphism and $L: \mathbb{R}^{2} \rightarrow \mathbb{R}^{2}$ represents various boundary conditions.

Problems of the existence/multiplicity of solutions to various BVPs (1.1), (1.2) have been investigated by numerous authors (see [1-6] and the references therein). They apply functional analytic methods (e.g., the Leray-Schauder approach) to get the existence of one solution and then, when the multiplicity is considered, the existence of the second one is proved by the lower/upper solutions techniques.

In the proposed approach, we consider the BVP

$$
\begin{aligned}
u^{\prime} & =\varphi^{-1}(v), \quad v^{\prime}=f\left(t, u, \varphi^{-1}(v)\right)-s, \\
L(z(t, c)) & =\left(L_{1}\left(u\left(t, c_{1}\right), v\left(t, c_{2}\right)\right), L_{2}\left(u\left(t, c_{1}\right), v\left(t, c_{2}\right)\right)\right)=0,
\end{aligned}
$$

equivalent to the BVP (1.1), (1.2) and are looking for the initial points of the $T$-periodic solutions of (1.3), which reduces the problem to finding zeros of the 
$\operatorname{mapping} \Psi: \mathbb{R}^{2} \rightarrow \mathbb{R}^{2}$

$$
\Psi(c)=L(z(t, c))=\left(L_{1}\left(u\left(t, c_{1}\right), v\left(t, c_{2}\right)\right), L_{2}\left(u\left(t, c_{1}\right), v\left(t, c_{2}\right)\right)\right),
$$

where

$$
\begin{aligned}
u\left(t, c_{1}\right) & =\int_{0}^{t} \varphi^{-1}\left(v\left(\sigma, c_{2}\right)\right) d \sigma+c_{1}, \\
v\left(t, c_{2}\right) & =\int_{0}^{t}\left(f\left(\sigma, u\left(\sigma, c_{1}\right), \varphi^{-1}\left(v\left(\sigma, c_{2}\right)\right)\right)-s\right) d \sigma+c_{2},
\end{aligned}
$$

satisfy the initial value problem $(1.3), z(0, c)=c=\left(c_{1}, c_{2}\right)$.

When the set of all zeros of $\Psi$ is known, the solution of (1.5) is determined using a corollary to Borsuk's theorem [8]. The searching of zeros of (1.5) and their multiplicities can be solved simultaneously, not referring to the theory of the upper/lower solutions, which considerably simplifies proofs and permits to get an extension of results of the quoted papers.

2. Preliminaries. Denote by $|\cdot|$ the norm in $\mathbb{R}^{2}$ and by $\|\cdot\|_{\infty}$ the maximum norm in $C^{0}(\mathbb{R}), x^{T}$ is the transpose to the vector $x . B\left(x_{0}, r\right)$ is the ball $\{x \in$ $\left.\mathbb{R}^{2}:\left|x-x_{0}\right|<r\right\}$. int $D, \operatorname{cl} D, \partial D$ stand for the interior, closure, and boundary of a set $D \in \mathbb{R}^{2}$, respectively. For $V \subset \mathbb{R}^{2}$, denote $S_{j}(V)=\{s \in \mathbb{R}:$ (1.3) has in cl $V$ at least $j ; T$-periodic solutions $\}$.

A pair $z(t, c)=\left(u\left(t, c_{1}\right), v\left(t, c_{2}\right)\right)$ is a solution of (1.3) provided it satisfies (1.3) a.e. for $t \in[0, T]$ and: $u \in C^{1}([0, T]), \varphi^{-1}\left(u^{\prime}\right)$ is absolutely continuous, $\left|u^{\prime}\right|<a$ if $\varphi:(-a, a,) \rightarrow \mathbb{R}$, or $|v|<a$ if $\varphi: \mathbb{R} \rightarrow(-a, a)$.

To simplify the presentation, assume additionally that the initial value problem (IVP) for (1.1) has a unique solution for any initial conditions. As it will be shown, this conditions may be removed.

The following theorem (see [8,3.31,Corollary] (stated here in a form slightly different from the quoted result; in [8] it is formulated for $d=0$ ) is basic.

Theorem B. Let $\Omega$ be a symmetric bounded open set in $\mathbb{R}^{n}$ with symmetry center at $d$ and $\psi: \operatorname{cl} \Omega \rightarrow \mathbb{R}^{n}$ be a continuous mapping never vanishing on $\partial \Omega$ such that for every $x \in \partial \Omega$,

$$
\alpha \psi(x-d) \neq(1-\alpha) \psi(d-x)
$$

for all $\alpha, 1 / 2 \leq \alpha \leq 1$.

Then $\psi(\Omega)$ contains a neighborhood of the origin.

Mappings $\Psi: \mathbb{R}^{2} \rightarrow \mathbb{R}^{2}$, associated with (1.4), corresponding to periodic or Neumann-Steklov boundary conditions and various right hand sides of (1.3) $g(t, u), h(t, u)$, or $f\left(t, u, \varphi^{-1} v(v)\right)$ are

$$
\Psi(c)=\left(\int_{0}^{T}\left(-g\left(u\left(\sigma, c_{1}\right)\right)+s\right) d \sigma, \int_{0}^{T} \varphi^{-1}\left(v\left(\sigma, c_{2}\right)\right) d \sigma\right)^{T},
$$




$$
\begin{aligned}
\Psi(c)= & \left(\int_{0}^{T}\left(\varphi^{-1}\left(v\left(\sigma, c_{2}\right)\right)-F\left(u\left(\sigma, c_{1}\right)\right)\right) d \sigma, \int_{0}^{T}\left(-h\left(u\left(\sigma, c_{1}\right)\right)+s\right) d \sigma\right)^{T} . \\
\Psi(c)= & \left(c_{2}-\varphi^{-1}\left(g_{0}\left(c_{1}\right)\right), \int_{0}^{T}\left(f\left(\sigma, u\left(\sigma, c_{1}\right), \varphi^{-1}\left(v\left(\sigma, c_{2}\right)\right)\right)-s\right) d \sigma\right. \\
& \left.+c_{2}-\varphi^{-1}\left(g_{T}\left(\int_{0}^{T} \varphi^{-1}\left(v\left(\sigma, c_{2}\right)+c_{1}\right)\right) d \sigma\right)\right)^{T} .
\end{aligned}
$$

The formula (2.3) represents the BVP

$$
\begin{aligned}
u^{\prime} & =\varphi^{-1}(v-F(u)), v^{\prime}=-h(t, u)+e(t)+s, \\
h(t, u) & =g(t, u) \text { or } a(t) q(u), \\
u(0)-u(T) & =v(0)-v(T)=0,
\end{aligned}
$$

equivalent to the periodic BVP for the Liénard-type equation

$$
\left(\varphi\left(u^{\prime}\right)\right)^{\prime}+f(u) u^{\prime}+h(t, u)=e(t)+s, \quad u(0)-u(T)=u^{\prime}(0)-u^{\prime}(T)=0 .
$$

\section{Results.}

Theorem 1 (see [1, Thm. 1]). If $\varphi:(a, a) \rightarrow \mathbb{R}, 0<a \leq \infty$, is an increasing homeomorphism, $\varphi(0)=0, g$ is continuous, and

$$
\begin{aligned}
& \int_{0}^{T} e(\sigma) d \sigma=0, \\
& g(u)>0 \quad \text { for all } u \in \mathbb{R}, \\
& \lim _{u \rightarrow \pm \infty} g(u)=0,
\end{aligned}
$$

then there exists an $s^{*} \in\left(0, \sup _{\mathbb{R}} g\right)$ such that the $B V P$

$$
\left(\varphi\left(u^{\prime}\right)\right)^{\prime}+g(u)=e(t)+s, \quad u(0)-u(T)=u^{\prime}(0)-u^{\prime}(T)=0,
$$

has zero, at least one, or at least two solutions according to $s \notin\left(0, s^{*}\right], s=s^{*}$, or $s \in\left(0, s^{*}\right)$.

Theorem 2 (see [1, Thm. 2]). Suppose $\varphi: \mathbb{R} \rightarrow(-a, a)$ with $0<a<\infty$ is an increasing homeomorphism, $\varphi(0)=0, g$ satisflies (3.1), (3.2), (3.3), and moreover

$$
\|e\|_{\infty} \leq\|g\|_{\infty}<\frac{a}{2 T},
$$

then there exists $s^{*} \in\left(0, \sup _{\mathbb{R}} g\right]$ such that the $B V P(3.4)$ has zero, at least one, or at least two solutions according to $s \notin\left(0, s^{*}\right), s=s^{*}$, or $s \in\left(0, s^{*}\right)$.

Remark 1. In contrast to $[1$, Thms. 1,2$], s^{*}$ can be chosen independently of $e(t)$. 
The presented method applies also in the search of $T$-periodic solutions to the Liénard-type equations (2.5).

Theorem 3 (see $[6$, Thms. 1.1, 1.2., 1.3]). Assume that $\varphi: \mathbb{R} \rightarrow \varphi(\mathbb{R})=\mathbb{R}$ is an increasing homeomorphism $\varphi(0)=0, f: \mathbb{R} \rightarrow \mathbb{R}$ is continuous, $h$ : $[0, T] \times \mathbb{R} \rightarrow \mathbb{R}$ is a Carathéodory function, so $h$ is continuous in u for a.a. $t \in[0, T]$, and is Lebesgue measurable in $t$ for all $u$. For $h(t, u)=a(t) g(u)$, the functions $a, e \in L^{\infty}[0, T]$ satisfy $a \geq 0$ with $\int_{0}^{T} a(\sigma) d \sigma=T, \int_{0}^{T} e(\sigma) d \sigma=0$.

Let one of the following group of conditions be satisfied:

(A) $\omega_{+}=\lim _{u \rightarrow \pm \infty} g(t, u)$ uniformly in $t \in[0, T]$ and $\omega_{+}=\infty$,

(B) $\omega_{ \pm}=\lim _{u \rightarrow \pm \infty} q(u)=\omega \in \mathbb{R}$ and $q(u)>\omega$ for $|u|>\rho$ sufficiently large,

(C) $\omega_{-}=+\infty$ and there is an $r$ such that $q(u)<\omega_{+} \in \mathbb{R}$ for $u \in(r,+\infty)$.

Then:

if (A) holds, there is an $s^{*} \in \mathbb{R}$ such that (2.5) has zero, at least one, or at least two T-periodic solutions according to $s<s^{*}, s=s^{*}$, or $s>s^{*}$.

if $(B)$ holds, then there is an $s^{*} \in(\omega, \infty)$ such that $(2.5)$ has zero, at least one, at least two T-periodic solutions according to $s>s^{*}, s=s^{*}, s \in\left(\omega, s^{*}\right)$. If $q(u)>\omega$ for all $u$, then (2.5) has no T-periodic solutions for $s \leq \omega$.

if (C) holds, set $\gamma=\omega_{+}$, then there are $\alpha, \beta$ such that

if $\min _{\{-\infty, r\}} g<\min _{\{r, \infty\}} g$, then $\beta<\alpha$ and (4.5) has no solution, at least one, at least two $T$-periodic solutions according to $s<\beta, s \in[\beta, \alpha) \cup[\gamma, \infty)$, or $s \in[\alpha, \gamma)$,

if $\min _{\{-\infty, r\}} g>\min _{\{r, \infty\}} g$, then $\alpha<\beta$ and (4.5) has no solution, at least one, at least two T-periodic solutions according to $s<\alpha, s \in[\alpha, \beta) \cup[\gamma, \infty)$, or $s \in[\beta, \gamma)$,

if $\min _{\{-\infty, r\}} g=\min _{\{r, \infty\}} g$, then $\alpha=\beta$ and (4.5) has no solution, at least one, at least two T-periodic solutions according to $s<\alpha, s \in\{\alpha\} \cup[\gamma, \infty)$, or $s \in(\beta, \gamma)$,

the condition $\min _{\{-\infty, r\}} g>\gamma$ implies $\gamma \leq \beta$ and (4.5) has at least one $T$-periodic solution for $s \in[\alpha, \gamma) \cup[\beta, \infty)$

Remark 2. Condition (C) $u \nearrow \omega_{+} \in \mathbb{R}$ is weakened to $u<\omega_{+} \in \mathbb{R}$, holding for $u$ large enough, moreover the conclusion of $(\mathrm{C})$ is extended.

Theorem 4 (see [3, Thm. 6]). Consider the BVP (1.1) with the NeumannSteklov boundary conditions

$$
v\left(0, c_{2}\right)-\varphi^{-1}\left(g_{0}\left(u\left(0, c_{1}\right)\right)\right)=0, \quad v\left(T, c_{2}\right)-\varphi^{-1}\left(g_{T}\left(u\left(T, c_{1}\right)\right)\right)=0 .
$$

Let $f:[0, T] \times \mathbb{R}^{2} \rightarrow \mathbb{R}$ be continuous and $\lim _{u \rightarrow \infty} f(t, u, v)=\infty$, uniformly with respect to $[0, T] \times(-a,, a), g_{0},-g_{T}: \mathbb{R} \rightarrow \mathbb{R}$ be continuous and bounded from below, $\varphi:(-a, a) \rightarrow \mathbb{R}, a<\infty, \varphi(0)=0$, be an increasing homeomorphism.

Then then there exists an $s^{*} \in \mathbb{R}$ such that the BVP for (1.1), (3.6) has zero, at least one, or at lest two solutions according to $s<s^{*}, s=s^{*}$, or $s>s^{*}$.

Remark 3. The conditions $g_{0}(0)=0, g_{0}(u) \geq 0$ for $u \geq 0$, and $g_{T}(0)=0$, $g_{T}(u) \leq 0$ for $u \geq 0$ are not needed. 
4. Proofs. By (3.2) and (3.3), one can assume $g(0)=\max \{g(u): u \in \mathbb{R}\}$. Theorems 1 and 2 deal with the BVP

$$
u^{\prime}=\varphi^{-1}(v), v^{\prime}=-g(u)+e(t)+s, \quad u(0)-u(T)=v(0)-v(T)=0,
$$

equivalent to (3.4). The periodicity conditions imply that $\Psi(c)$ defined by $(2.2)$ has a root.

Proof of Theorem 1. From (2.2), it follows that for $s>g(0)$, the BVP has no $T$-periodic solutions. For $0<s \leq g(0)$, from (3.2), we get

$$
\begin{aligned}
\left|v\left(t, c_{2}\right)-c_{2}\right| & \leq \int_{0}^{T}\left(\left|-g\left(u\left(\sigma, c_{1}\right)\right)+s\right|+|e(\sigma)|\right) d \sigma \\
& <\int_{0}^{T}\left(g(0)+\|e\|_{\infty}\right) d \sigma=b T .
\end{aligned}
$$

Since $v \varphi^{-1}(v)>0$ for $v \neq 0$, we have $c_{2} \varphi^{-1}\left(v\left(t, c_{2}\right)\right)>0$ for $t \in[0, T]$ and $\left|c_{2}\right|>b T$, which gives

$$
c_{2} \int_{0}^{T} \varphi^{-1}\left(v\left(\sigma, c_{2}\right)\right) d \sigma>0 \quad \text { for } c_{1} \in \mathbb{R},\left|c_{2}\right|>b T .
$$

Let $a^{*}=\max \left\{\left|\varphi^{-1}(-b T)\right|, \varphi^{-1}(b T)\right\}$, then by the first formula (1.6), $c_{1}-$ $a^{*} T \leq u\left(t, c_{1}\right) \leq c_{1}+a^{*} T$ and for $s_{0}<\min \left\{g(u): u \in\left[-a^{*} T, a^{*} T\right]\right\}$, we obtain

$$
\int_{0}^{T}\left(-g(u(\sigma, 0))+s_{0}\right) d \sigma<0 \text { for }\left|c_{2}\right| \leq b T .
$$

For $s_{0} \in(0, g(0))$, there is an $N\left(s_{0}\right)$ such that $-g(u)+s_{0}>0$ for $|u|>$ $N\left(s_{0}\right)$. Hence $-g\left(u\left(t, c_{1}\right)\right)+s_{0}>0$ for $t \in[0, T],\left|c_{1}\right| \geq N\left(s_{0}\right)+a^{*} T$, and $\left|c_{2}\right| \leq b T$, implying that

$$
\int_{0}^{T}\left(-g\left(u\left(\sigma, c_{1}\right)\right)+s_{0}\right) d \sigma>0 \text { for }\left|c_{1}\right| \geq N\left(s_{0}\right)+a^{*} T \text { and }\left|c_{2}\right| \leq b .
$$

By (4.3), (4.4), (4.5), the mapping (2.2) does not vanish on the boundaries the bounded symmetric sets $U_{2}\left(s_{0}\right)=\left(-\left(N\left(s_{0}\right)+a^{*} T\right), 0\right) \times(-b T, b T)$, $\left.U_{1}\left(s_{0}\right)=\left(0, N\left(s_{0}\right)+a^{*} T\right)\right) \times(-b T, b T)$ and Theorem B shows $\Psi\left(c^{i}\left(s_{0}\right)\right)=0$ for $c^{i}\left(s_{0}\right) \in U_{i}\left(s_{0}\right)$ and $S_{1}\left(U_{1}\left(s_{0}\right)\right)=S_{1}\left(U_{2}\left(s_{0}\right)\right)$.

Let $s^{*}=\sup \left\{S_{1}\left(U_{1}\left(s_{0}\right)\right): 0<s_{0}<g(0)\right\}$. From $S_{1}\left(U_{1}\left(s_{0}\right)\right)=S_{1}\left(U_{2}\left(s_{0}\right)\right)$, since $s_{0}>0$ has been arbitrarily chosen, we get $S_{2}\left(U_{1}\left(s^{*}\right) \cup U_{2}\left(s^{*}\right)\right)=\left(0, s^{*}\right)$, proving the existence of at least two different solutions $z=z\left(t, c^{i}(s)\right)(i=$ $1,2)$ of $(4.1)$ for $s \in\left(0, s^{*}\right)$. Let $\{s(k)\}$ be an increasing sequence satisfying $\lim _{k \rightarrow \infty} s(k)=s^{*}$ and let $\left\{z\left(t, c^{1}(s(k))\right\}\right.$ be the corresponding $T$-periodic solution of (4.1). 
For all $k, U_{1}(s(k)) \subset \operatorname{cl} U_{1}(s(0))$ hence passing, if necessary, to a subsequence, we conclude that $\lim _{k \rightarrow \infty} z\left(t, c^{1}(s(k))\right)$ is $T$-periodic, which completes the proof.

Proof of Theorem 2. By (3.5), $g(0)+\|e\|_{\infty}<2 g(0)=b / T$ with $b<a$ and $\left|v\left(t, c_{2}\right)-c_{2}\right| \leq \int_{0}^{T}(2 g(0) d \sigma \leq b$ for $t \in[0, T]$.

If the component $v\left(t, c_{2}\right)$ of $z(t, s)$ is $T$-periodic, then

$$
c_{2}-b \leq v\left(t, c_{2}\right) \leq c_{2}+b \text { for } t \in[0, T], c_{2} \in \mathbb{R},
$$

independent of the choice of an increasing homeomorphism $\varphi$, assuming only that $\varphi(0)=0$.

For the proof, note that $u^{\prime}\left(\xi, c_{1}\right)=0$ for a certain $\xi \in[0, T]$ which by (4.1) implies that $v\left(\xi, c_{2}\right)=0$ and from $\left|v\left(t, c_{2}\right)-v\left(\xi, c_{2}\right)\right|=\left|v\left(t, c_{2}\right)\right| \leq b$, the formula (4.6) follows.

Following [1], set $p=\max \left\{\left|\varphi^{-1}( \pm b)\right|\right\}$ and for an increasing homeomorphism $\psi: \mathbb{R} \rightarrow \mathbb{R}$ satisfying $\left.\psi\right|_{[-p, p]}=\varphi$, define the modification of (4.1)

$$
u^{\prime}=\psi^{-1}(v), \quad v^{\prime}=-g(u)+s+e(t)
$$

having the same $T$-periodic solutions as (4.1).

From $v \psi(v)>0, v \neq 0$, and (4.6), we get that (4.3) is valid for $\left|c_{2}\right| \geq M>b$. The solution of (4.7) satisfies for all $t \in[0, T], c_{2} \in[-M, M]$, the inequality

$$
\left|u\left(t, c_{1}\right)-c_{1}\right|=\left|\int_{0}^{T} \varphi^{-1}\left(v\left(\sigma, c_{2}\right)\right) d \sigma\right| \leq p T .
$$

If $s<m=\min \{g(u): u \in[-p T, p T]\}$ and $c_{2} \in[-M, M]$, then

$$
\int_{0}^{T}(-g(u(\sigma, 0))+s) d \sigma<0 .
$$

As in Theorem 1, by (4.8), (4.9), for a fixed $0<s_{0}<m$, there is an $N\left(s_{0}\right)>0$ such that for $\left|c_{1}\right| \geq N\left(s_{0}\right)+p T$ and $c_{2} \in[-M, M]$, the inequality (4.5) holds.

From the inequalities (4.9), (4.5), and (4.3), it follows that $\Psi$ in each of the sets

$$
\begin{aligned}
& U_{2}\left(s_{0}\right)=\left(-\left(N\left(s_{0}\right)+p T\right), 0\right) \times(-M, M), \\
& U_{1}\left(s_{0}\right)=\left(0, N\left(s_{0}\right)+p T\right) \\
& \quad \times(-M, M)
\end{aligned}
$$

fulfills the conditions of Theorem B, proving that $S_{2}\left(U_{1} \cup U_{2}\right)$ is nonempty. The remaining part repeats the argument of the previous proof.

Proof of Theorem 3. Observe that for each of the conditions (A)-(C), there is an $s_{0}$ and the corresponding $\rho(s)>0$ such that for $|u|>\rho(s)$,

$$
\varepsilon \min \left\{-h(t, u)+s_{0}: \text { a.a. } t \in[0, T]\right\}>0 \quad(\varepsilon=-1 \text { or } 1)
$$

with $\varepsilon=-1$ in cases (A), (C) or $\varepsilon=1$ in the case (B). In cases (A) and (B), one can assume that $g(0)=\min \{g(u): u \in \mathbb{R}\}$. 
We begin with lemmas.

Lemma 1. Assume that for $s_{0} \in \mathbb{R}$, under one of the conditions $(A)-(C),(2.5)$ has a T-periodic solution $z(t, c)=\left(u\left(t, c_{1}\right), v\left(t, c_{2}\right)\right)$, then there is a constant $M\left(s_{0}\right)$ independent of $c$ such that $u\left(t, c_{1}\right)$ satisfies

$$
\left|u\left(t, c_{1}\right)\right|<M\left(s_{0}\right) \quad \text { for a.a. } t \in[0, T] .
$$

Proof of Lemma 1. Suppose (4.11) does not hold. Let $\left\{z\left(t, c_{n}\right)\right\}$ be the sequence of $T$-periodic solutions to (2.5) satisfying $\lim _{n \rightarrow \infty}\left\|u\left(\cdot, c_{1 n}\right)\right\|_{\infty}=\infty$.

As $u\left(t, c_{1 n}\right)$ are $T$-periodic, they do not satisfy (4.10) for all $t \in[0, T]$ and for a certain $\tau\left(c_{1 n}\right) \in[0, T], \varepsilon\left(-h\left(\tau_{1 n}, u\left(\tau, c_{1 n}\right)+s_{0}\right) \leq 0\right.$, hence $\left\{\left(\tau\left(c_{1 n}\right)\right)\right.$, $\left.\left.u\left(\tau\left(c_{1 n}\right), c_{1 n}\right)\right)\right\}$ is contained in the compact set $A=[0, T] \times\left[-\rho\left(s_{0}\right), \rho\left(s_{0}\right)\right]$ and it has a subsequence $\left\{\left(t_{n(k)}, u_{n(k)}\right)\right\}$ converging to $\left(t_{0}, d_{1}\right) \in A$ corresponding to the $T$-periodic function $u\left(\cdot, d_{1}\right)$ contradicting $\lim _{k \rightarrow \infty}\left\|u\left(\cdot, c_{1 n(k)}\right)\right\|_{\infty}=\infty$.

Lemma 2. If $z(t, c)$ is the T-periodic solution of (2.5), then from (4.11) it follows that for $t \in[0, T]$ and a given $s_{0}$, one has the inequalities

$$
\begin{aligned}
& \left|v\left(t, c_{2}\right)-c_{2}\right| \leq m\left(s_{0}\right) T, \quad\left|v\left(t, c_{2}\right)-c_{2}-F\left(u\left(t, c_{1}\right)\right)\right| \leq P\left(s_{0}\right), \\
& \left|u\left(t, c_{1}\right)-c_{1}\right| \leq m_{1}\left(s_{0}\right) T,
\end{aligned}
$$

where constants $m\left(s_{0}\right), N\left(s_{0}\right), m_{1}\left(s_{0}\right)$ are defined by

$$
\begin{aligned}
m\left(s_{0}\right) & =\max \left\{\left|g(t, u)-s_{0}+e(t)\right|: \text { a.a. } t \in[0, T],|u| \leq M\left(s_{0}\right)\right\}, \\
N\left(s_{0}\right) & =\max \left\{|F(u)|:|u| \leq M\left(s_{0}\right)\right\}, P\left(s_{0}\right)=m\left(s_{0}\right) T+N\left(s_{0}\right), \\
m_{1}\left(s_{0}\right) & =\varphi^{-1}\left(P\left(s_{0}\right)\right) .
\end{aligned}
$$

Proof of Lemma 2. By the formula $\left|v^{\prime}\left(t, c_{2}\right)\right|=\left|g\left(t, u\left(t, c_{1}\right)\right)-s_{0}+e(t)\right| \leq$ $\left.m\left(s_{0}\right)\right)$, we get two first inequalities. The last one results from $(4.12), \mid v\left(t, c_{2}\right)-$ $F\left(u\left(t, c_{1}\right)\right) \mid \leq P\left(s_{0}\right)$, and $\left|u\left(t, c_{1}\right)-c_{1}\right|=\left|\int_{0}^{t} \varphi^{-1}\left(v\left(\sigma, c_{2}\right)-F\left(u\left(\sigma, c_{1}\right)\right)\right) d \sigma\right|$.

Passing to the proof of the theorem, assume the case (A).

Let $h(t, u)=g(t, u)$. By $(2.3)$, the $T$-periodic solutions $z(t, c)$ to $(2.5)$ are possible for $s>\min \{g(t, u): T \in[0, T], u \in \mathbb{R}\}$ and the component $u\left(t, c_{1}\right)$ of $z(t, c)$ satisfies $(4.11)$.

Choose $s_{0}>m_{A}=\max \left\{g(t, u): T \in[0, T],|u| \leq M\left(s_{0}\right)\right\}$. Then

$$
\int_{0}^{T}\left(-g(\sigma, u(\sigma, 0))+s_{0}\right) d \sigma>0 .
$$

From (4.12), we have for $\left|c_{2}\right|>P\left(s_{0}\right)$ and $c_{1} \in \mathbb{R}$,

$$
c_{2} \int_{0}^{T} \varphi^{-1}\left(v\left(\sigma, c_{2}\right)-F(u(\sigma, 0))\right) d \sigma>0 .
$$

If $\left|c_{2}\right| \leq P\left(s_{0}\right)$, then from the last formula (4.12) and the inequality

$$
s_{0}<\min \left\{g(t, u): t \in[0, T],|u|>\rho\left(s_{0}\right)\right\}
$$


holding for $\rho\left(s_{0}\right)$ large enough, it follows that

$$
\int_{0}^{T}\left(-g\left(\sigma, u\left(\sigma, c_{1}\right)\right)+s_{0}\right) d \sigma<0 \text { for }\left|c_{1}\right|>m_{1}\left(s_{0}\right) T+\rho\left(s_{0}\right) .
$$

Conditions (4.14), (4.15), and (4.16) define boundaries of sets

$$
\begin{aligned}
& U_{1}\left(s_{0}\right)=\left(-\rho\left(s_{0}\right)-m_{1}\left(s_{0}\right) T, 0\right) \times\left(-P\left(s_{0}\right), P\left(s_{0}\right)\right), \\
& U_{2}\left(s_{0}\right)=\left(0, \rho\left(s_{0}\right)+m_{1}\left(s_{0}\right) T\right) \times\left(-P\left(s_{0}\right), P\left(s_{0}\right)\right) .
\end{aligned}
$$

$\Psi(c)$ considered on each of them fulfills the assumptions of Theorem B, hence (2.3) for $s=s_{0}$ has at least two distinct roots, showing that $S_{2}\left(U_{1}\left(s_{0}\right) \cup\right.$ $\left.U_{2}\left(s_{0}\right)\right) \neq \emptyset$.

Let $s^{*}=\inf \left\{s_{0}: S_{2}\left(U_{1}\left(s_{0}\right) \cup U_{2}\left(s_{0}\right)\right)>0\right\}$. By the argument of Theorem 1, the system (2.5) with $s=s^{*}$ has at least one solution.

Case (B). If for $s_{0}, z(t, c)$ is periodic, then Lemma 1 implies that its component $u\left(t, c_{1}\right)$ fulfills (4.11). $T$-periodic solutions to (2.5) are possible for $s \in K=$ $\left(n_{B}, m_{B}\right)$, where $n_{B}=\min \{g(u): u \in \mathbb{R}\}$ and $m_{B}=\max \left\{q(u):|u| \leq M\left(s_{0}\right)\right\}$.

Fix $s_{0} \in\left(\omega, m_{B}\right)$. Then for all $c_{2} \in \mathbb{R}$,

$$
\int_{0}^{T}\left(-a(\sigma) q(u(\sigma, 0))+s_{0}\right) d \sigma<0 .
$$

From (4.10) and (4.12), for $\left|c_{1}\right| \geq m_{1}\left(s_{0}\right) T+\rho\left(s_{0}\right)$, we get

$$
\int_{0}^{T}\left(-a(\sigma) q(u(\sigma, 0))+c_{1}\right) d \sigma>0 .
$$

By (4.19), (4.18), (4.15), and Theorem B, (2.5) has a solution at each of the sets $(4.17)$, hence $S_{2}\left(U\left(s_{0}\right)\right)=S_{1}\left(U_{1}\left(s_{0}\right) \cup U_{2}\left(s_{0}\right)\right)=\left(\omega, s^{*}\right)$, where $s^{*}=$ $\sup \left\{S_{2}\left(U\left(s_{0}\right)\right): s_{0} \leq R_{+}\right\}$.

For $s^{*}$, repeating the argument of Theorem 1, one shows that (2.5) has at least one $T$-periodic solution.

If $u>\omega$ for all $u$, then $\int_{0}^{T}\left(-h\left(u\left(\sigma, c_{1}\right)\right)+s\right) d \sigma>0$ for $s<\omega$ implying the last assertion.

Case (C). The $T$-periodic solutions may occur for $s>m=\min \{a(t) q(u)$ : $T \in[0, T], u \in \mathbb{R}\}$. By (4.10), and Lemma 1 , if for $s_{0}>m$ the solution $z(t, c)$ is $T$-periodic, then $\left|u\left(t, c_{1}\right)\right| \leq M\left(s_{0}\right)$.

Define $m_{C}=\max \left\{q(u) a(t):|u| \leq M\left(s_{0}\right)\right.$, a.a. $\left.t \in[0, T]\right\}$.

For $c_{0}>r+M\left(s_{0}\right)$, a.a. $t \in[0, T]$, and $s_{0} \in\left(m_{C}, \omega_{+}\right)$, we get $-a(t) q\left(u\left(c_{0}\right)\right)$ $+s_{0}>0$, implying

$$
\int_{0}^{T}\left(-a(\sigma) q\left(u\left(\sigma, c_{0}\right)\right)+s_{0}\right) d \sigma>0
$$


and, by Remark 2, the reversed inequality

$$
\int_{0}^{T}\left(-a(\sigma) q\left(u\left(\sigma, c_{1}\right)\right)+s_{0}\right) d \sigma<0,
$$

holding for $\left|c_{1}\right|>\rho\left(s_{0}\right)+m_{1}\left(s_{0}\right) T$.

With $N\left(s_{0}\right), P\left(s_{0}\right), m_{1}\left(s_{0}\right)$ defined as in Lemma 2, we get inequality (4.15) which together with (4.20), (4.21) describes the sets

$$
\begin{aligned}
& U_{1}\left(s_{0}\right)=\left(-\rho\left(s_{0}\right)-m_{1}\left(s_{0}\right) T, c_{0}\right) \times\left(-P\left(s_{0}\right), P\left(s_{0}\right)\right), \\
& U_{2}\left(s_{0}\right)=\left(c_{0}, \rho\left(s_{0}\right)+m_{1}\left(s_{0}\right) T\right) \times\left(-P\left(s_{0}\right), P\left(s_{0}\right)\right) .
\end{aligned}
$$

In each set $U_{1}, U_{2}$, by Theorem $\mathrm{B}, \Psi(c)=0$ has a solution.

Setting $\alpha=\inf S_{1}\left(U_{1}\right), \beta=\inf S_{1}\left(U_{2}\right), \gamma=\omega_{+}$, we have

$S_{1}\left(U_{1} \cup U_{2}\right)$, and $S_{2}\left(U_{1} \cup U_{2}\right)=\left\{\begin{array}{l}{[\beta, \alpha] \cup[\gamma, \infty), \text { and }(\alpha, \gamma) \text { for } \beta<\alpha} \\ {[\alpha, \beta] \cup[\gamma, \infty), \text { and }(\alpha, \gamma) \text { for } \alpha<\beta} \\ \{\beta\}, \cup[\gamma, \infty), \text { and }(\beta, \gamma) \text { for } \beta=\alpha \\ {[\alpha, \gamma) \cup[\beta, \infty), \quad \text { and } \emptyset \text { for } \beta>\gamma .}\end{array}\right.$

By the argument of Theorem 1, one shows that the points $\alpha, \beta$ are the initial points of the $T$-periodic solutions.

Proof of Theorem 4. The functions $f(t, u, v), \varphi^{-1}\left(g_{0}(u)\right),-\varphi^{-1}\left(g_{T}(u)\right)$ are bounded from below, hence for $s<f_{D}=\min \left\{f(t, u, v)+\varphi^{-1}\left(g_{0}(u)\right)-\varphi^{-1}\right.$ $\left.\left.\left(g_{T}(u)\right): u \in \mathbb{R}\right\},|v|<a\right\}$, the mapping (1.3) has no zeros.

Take $s_{0}>f_{D}$ such that

$$
\begin{aligned}
& \int_{0}^{T}\left(f\left(t, u(\sigma, 0), \varphi^{-1}(v(\sigma, 0))\right)-s_{0}\right) d \sigma \\
& \quad+g_{0}(u(0,0))-g_{T}(u(T, 0))<0 .
\end{aligned}
$$

By the inequality $\left|u\left(t, c_{1}\right)-c_{1}\right| \leq a T$, for any fixed $s_{0}>f_{D}$, there is an $M\left(s_{0}\right)$ such that for $\left|c_{1}\right|>M\left(s_{0}\right)$,

$$
\begin{aligned}
& \int_{0}^{T}\left(f\left(\sigma, u\left(\sigma, c_{1}\right), \varphi^{-1}\left(v\left(\sigma, c_{2}\right)\right)\right)-s_{0}\right) d \sigma \\
& \quad+g_{0}\left(u\left(0, c_{1}\right)\right)-g_{T}\left(u\left(T, c_{1}\right)\right)>0 .
\end{aligned}
$$

Let $m\left(s_{0}\right)=\max \left\{g_{0}\left(u\left(0, c_{1}\right)\right): c_{1} \in\left[-M\left(s_{0}\right), M\left(s_{0}\right)\right]\right\}$. Then for $\left|c_{2}\right|>$ $m\left(s_{0}\right)$,

$$
c_{2}\left(v\left(0, c_{2}\right)-g_{0}\left(u\left(0, c_{1}\right)\right)\right)>0 .
$$

From $(4.23),(4.24),(4.25)$, it follows that the mapping (2.4) considered in the sets

$$
\begin{aligned}
& U_{1}\left(s_{0}\right)=\left(-M\left(s_{0}\right), 0\right) \times\left(-m\left(s_{0}\right), m\left(s_{0}\right)\right), \\
& U_{2}\left(s_{0}\right)=\left(0, M\left(s_{0}\right)\right) \times\left(-m\left(s_{0}\right), m\left(s_{0}\right)\right),
\end{aligned}
$$


satisfies the conditions of Theorem $\mathrm{B}$ and for $s_{0}>f_{D}$, has the root in each set $U_{i}(s)$, proving thus the existence of at least two different $T$-periodic solutions $z\left(t, c^{i}\left(s_{0}\right)\right), i=1,2$, of the BVP (1.3), (3.6)

Proceeding as in the proof of Theorem 1 , let $s^{*}=\inf \left\{S_{1}\left(U_{i}(s)\right): s \in \mathbb{R}, i=\right.$ $1,2\}$ and set $c^{i}=\lim _{s \rightarrow s^{*}} c^{i}(s)$. Then the corresponding solutions $z\left(t, c^{i}\right)$, $i=1,2$, are $T$-periodic and satisfy $\Psi\left(c^{i}\right)=0$ showing the second part of Theorem 4.

If $c^{1}=c^{2}$, from the uniqueness of the solution to (1.3), we get the existence of exactly one solution of the BVP (1.3), (3.6) for $s=s^{*}$.

This completes the proof of Theorem 4 in the case of the unique IVPs.

Remark 4. Theorems 1-4 hold also in the lack of the uniqueness of the IVP for (1.1).

Proof. Let $K$ be the compact set containing in int $K$ all solutions of a considered IVP for $[0, T]$ and $c \in \operatorname{cl}\left(U_{1} \cup U_{2}\right)$.

Approximate (see $\left[7\right.$, Ch. 1, Thm. 2.4]) $\varphi^{-1}$ and $f$ uniformly on $[0, T] \times K$ by smooth functions $g_{k}, f_{k}(k=1,2, \ldots)$ such that $g_{k}$ is a homeomorphism of $\mathbb{R}^{2}$ onto the ball $B\left(0, a_{n}\right)$ with $g_{k}\left(p_{k}\right)=0,\left(\lim _{k \rightarrow \infty}\left(p_{k}, a_{k}\right)=(0, a)\right)$ and $f_{k}$ fulfilling the assumptions of a considered theorem.

The system

$$
u^{\prime}=g_{k}(v), \quad v^{\prime}=h_{k}(t, u, v)
$$

approximating in $K$ the considered theorem has the uniqueness property of the IVP for $k>k_{0}$. Apply Theorems $1-4$ to get solutions $z_{k}\left(t, c^{k}\right)$ of the corresponding theorems. Since $\left\{z_{k}\left(t, c^{k}\right)\right\}$ are in $K$, by the Ascoli theorem, they contain subsequences uniformly converging to solutions described in Theorems $1-4$.

Open Access. This article is licensed under a Creative Commons Attribution 4.0 International License, which permits use, sharing, adaptation, distribution and reproduction in any medium or format, as long as you give appropriate credit to the original author(s) and the source, provide a link to the Creative Commons licence, and indicate if changes were made. The images or other third party material in this article are included in the article's Creative Commons licence, unless indicated otherwise in a credit line to the material. If material is not included in the article's Creative Commons licence and your intended use is not permitted by statutory regulation or exceeds the permitted use, you will need to obtain permission directly from the copyright holder. To view a copy of this licence, visit http://creativecommons. org/licenses/by/4.0/.

Publisher's Note Springer Nature remains neutral with regard to jurisdictional claims in published maps and institutional affiliations.

\section{References}

[1] Bereanu, C., Mawhin, J.: Multiple periodic solutions of ordinary differential equations with bounded nonlinearities and $\varphi$-Laplacian. Nonlinear Differential Equations Appl. NoDEA 15, 159-168 (2008) 
[2] Bereanu, C., Mawhin, J.: Existence and multiplicity results for some nonlinear problems with singular $\phi$-Laplacian. J. Differential Equations 243, 536-555 (2007)

[3] Bereanu, C., Mawhin, J.: Nonhomogeneous boundary value problems for some nonlinear equations with singular $\phi$-Laplacian. J. Math. Anal. Appl. 352, 218233 (2009)

[4] Bereanu, C., Mawhin, J.: Boundary value problems for some nonlinear systems with singular $\varphi$-Laplacian. J. Fixed Point Anal. Appl. 4, 37-75 (2008)

[5] Cid, J.A., Sanches, L.: Periodic solutions for second order differential equations with discontinuous restorting forces Math. Anal. Appl 288, 349-364 (2003)

[6] Feltrin, G., Sovrano, E., Zanolin, F.: Periodic solutions to parameter-dependent equations with $\phi$-Laplacian type operator. Nonlinear Differential Equations Appl. NoDEA 26, 26-38 (2019)

[7] Hartman, P.: Ordinary Differential Equations. Academic Press, New York (1964)

[8] Schwartz, J.T.: Nonlinear Functional Analysis. Gordon and Breach, New York (1969)

[9] Ward, J.R.: Periodic solutions of ordinary differential equations with bounded nonlinearities. Topol. Methods Nonlinear Anal. 19, 275-282 (2002)

StanisŁaW SȨDZIWY

Theoretical Computer Science, Faculty of Mathematics and Computer Science Jagiellonian University

ul. Łojasiewicza 6

30-348 Kraków

Poland

e-mail: sedziwy@ii.uj.edu.pl

Received: 28 May 2021

Accepted: 11 September 2021. 